\title{
ANSIEDADE NO CLIMATÉRIO: PREVALÊNCIA E FATORES ASSOCIADOS
}

\author{
CLIMACTERIC IN ANXIETY: PREVALENCE \\ AND ASSOCIATED FACTORS
}

Wendry Maria Paixão Pereira ${ }^{1}$

Ana Carolina Basso Schmitt ${ }^{2}$

Cássia Maria Buchalla ${ }^{3}$

Alberto Olavo Advincula Reis ${ }^{4}$

José Mendes Aldrighi ${ }^{5}$

Pereira WMP, et al. Ansiedade no climatério: prevalência e fatores associados. Rev Bras Crescimento Desenvolvimento Hum. 2009; 19(1): 89-97.

\section{Resumo:}

Este estudo estimou a prevalência de ansiedade e caracterizou os possíveis fatores associados em 749 mulheres no climatério, cadastradas no Programa de Saúde da Família e integrantes do Projeto de Saúde de Pindamonhangaba (PROSAPIN). Trata-se de um estudo observacional transversal; o método consistiu na aplicação de um questionário auto-referido, contendo variáveis sócio-demográficas, de ansiedade e antropometria. A análise estatística foi feita pela distribuição de freqüências e Odds Ratio (intervalo de confiança de 95\%), considerando o nível de significância em $\mathrm{p}<0,05$. Os resultados mostraram que a prevalência de ansiedade foi de 49,8\% (IC 95\%: 46,2 a 53,4) e, entre os principais fatores associados à ansiedade destacaram-se a escolaridade (OR:1,20 p=0,005), atividade remunerada (OR:0,68 $\mathrm{p}=0,001)$ e o período da transição menopausal (OR:1,21 p=0,003). Em conclusão, a ansiedade foi muito prevalente, atingindo quase metade da população estudada e os principais fatores associados à ansiedade foram os hábitos de vida e as condições socioeconômicas.

Palavras-chave: ansiedade; climatério; menopausa; pós-menopausa.

1 Mestranda do Departamento de Saúde Materno-Infantil da Faculdade Saúde Pública / USP e Bolsista FAPESP, Processo nº 08/ 52607-8

2 Doutora pela Faculdade de Saúde Pública / USP

3 Professora Doutora do Departamento de Epidemiologia da Faculdade de Saúde Pública / USP

4 Professor Doutor do Departamento Saúde Materno-Infantil da Faculdade de Saúde Pública / USP

5 Professor Associado Departamento Saúde Materno-Infantil da Faculdade de Saúde Pública / USP

Correspondência para: Wendry Maria Paixão Pereira. Rua: Dr João Dias Cardoso Sobrinho,36. Jardim Maria Augusta - Taubaté / SP. Cep: 12070-010.

E-mail: wendrypaixao@usp.br 


\begin{abstract}
:
The present study estimated the prevalence of anxiety and characterized the possible associated factors in 749 women in menopause, registered in the Family Health Program and members of the Project of Health Pindamonhangaba (PROSAPIN). This is a cross sectional observational study, the method was the application of a self-reported questionnaire containing socio-demographic variables of anxiety and anthropometry. Statistical analysis was performed by the distribution of frequencies and Odds Ratio (confidence interval=95\%), considering the level of significance at $\mathrm{p}>0.05$. The results showed that the prevalence of anxiety was $49.8 \%$ (95\% CI: 46.2 to 53.4 ) and, among the main factors associated with the anxiety of such a school (OR:1,20 p=0.005), activity (OR:0.68 p=0.001) and duration of menopausal transition (OR:1.21 p=0.003). In conclusion, the anxiety was very prevalent, affecting almost half the population studied and the main factors associated with anxiety were the living habits and socioeconomic.
\end{abstract}

Key words: anxiety; climacteric; menopause; postmenopause.

\section{INTRODUÇÃO}

O climatério representa a fase de transição entre o estado reprodutivo e o não reprodutivo e Notelovitz ${ }^{1}$ caracterizou didaticamente seu início aos 35 e término aos 65 anos. Para Soules et al. ${ }^{2}$, constitui-se de pequenas alterações no ciclo menstrual que caracterizam o início do climatério.

Para Gracia et al. ${ }^{3}$, o período climatério advém do envelhecimento ovariano (Penn Ovarian Aging Study), sendo constituído por cinco estágios: fetal, infantil, reprodutivo, transição menopausal e pós menopausa. Além de serem facilmente identificados clinicamente, permitem entender com mais precisão o início etário do climatério, caracterizando os estágios da transição menopausal e pós-menopausa.

O envelhecimento ovariano representa um processo fisiológico e contínuo com início a partir de 20 semanas de gestação, quando o número de folículos é de sete milhões.

A partir daí, ocorre progressivo consumo folicular, atingindo por ocasião da primeira menstruação (menarca) 400.000 unidades; em torno dos 36-37 anos, 25.000 unidades. Esta é a fase de inicio do estágio da transição menopausal precoce, caracterizada por irregularidades menstruais, decorrentes de mínimas alterações na produção de hormônios produzidos pelos ovários - o estrogênio e a progesterona. ${ }^{4}$

Assim, com a progressão do envelhecimento ovariano instala-se a transição menopausal tardia, manifestada por acentuado hipoestrogenismo e evidentes alterações menstruais, como amenorréia de 3 a 11 meses. Ao exceder 12 meses de amenorréia consecutivos ${ }^{5}$ firma-se o diagnóstico retrospectivo de menopausa.

Com a falência ovariana há uma baixa significativa do estrogênio que resulta na presença de sintomas desconfortáveis, com destaque para as ondas de calor, insônia, atrofia vaginal, depressão e ansiedade. , $7,8^{\circ}$

Dentre estes sintomas, a ansiedade pode se manifestar pela presença da irritabilidade, taquicardia, sudorese, fadiga e preocupação excessiva com pequenos problemas. ${ }^{9}$

Desta maneira, considera-se que a ansiedade corrobora para alteração na qualidade de vida. O objetivo do estudo é estimar a prevalência de ansiedade e caracterizar seus 
fatores associados em mulheres nos estágios da transição e da pós menopausa.

\section{MÉTODO}

Realizou-se estudo observacional transversal no período de outubro de 2007 a janeiro de 2008, vinculado ao Projeto de Saúde de Pindamonhangaba (PROSAPIN). O projeto obteve aprovação do Comitê de Ética da Faculdade Saúde Publica, protocolo n ${ }^{0} 1776$ da Universidade de São Paulo.

Foram selecionadas aleatória e sistematicamente 875 mulheres na transição menopausal e pós-menopausa, cadastradas no Programa de Saúde da Família (PSF), totalizando assim 749 mulheres e divididas em três grupos: Dos 35 aos 44 anos ( $\mathrm{N}=307)$, do 45 aos 54 anos $(\mathrm{N}=265)$ e dos 55 aos 65 anos ( $\mathrm{N}=163)$, das quais 14 mulheres não responderam sobre a idade. Estas divisões são correspondentes a três décadas do climatério: inicial, intermediário e tardio. ${ }^{1}$

As mulheres portadoras de alterações cognitivas e/ou neurológicas não compuseram o estudo. As mulheres incluídas no protocolo de pesquisa foram recrutadas em seus domicílios por agentes comunitários de saúde. Todas as participantes foram entrevistadas por staffs treinados; o tempo médio da entrevista foi de uma hora e trinta minutos.

Depois da entrevista, as mulheres foram avaliadas por uma equipe de graduandos de fisioterapia, treinada pelo Laboratório de Avaliação Nutricional de Populações da Faculdade Saúde Publica da Universidade de São Paulo que coletaram os dados antropométricos: circunferências do abdômen e do quadril, peso e altura, sempre no período da manhã.

Outro instrumento utilizado foi o questionário. Composto de dados pessoais, socioeconômicos, história ginecológica, morbidade, hábitos de vida, atividade física e ansiedade.

Já o instrumento para avaliação da ansiedade foi o Inventário de Ansiedade de BECK (BAI), validado por Cunha ${ }^{11}$ e composto de 21 itens, cada um com uma escala de quatro opções de respostas e com graus ou estágios de gravidade crescente de ansiedade: mínimo de 0 a 10, leve de 11 a 19, moderado de 20 a 30 e grave de 31 a 63. Foi considerada ansiedade clinicamente importante a partir do estágio leve.

Como controle da coleta dos dados, foi realizado ao longo do dia e ao final do estudo, $10 \%$ de reavaliações aleatórias na população estudada, na forma de re-entrevista, por outro membro do grupo de pesquisa. Os dados obtidos foram comparados com as entrevistas originais para avaliar sua qualidade, visando a identificar eventuais imprecisões e possíveis erros sistemáticos. Os dados foram digitados duplamente para redução dos possíveis vieses.

Para este estudo foram analisados os seguintes fatores associados à ansiedade: idade, renda, anos de estudo, raça, religião, estado civil, ocupação, uso de terapia hormonal, morbidade associada, tabagismo, uso de álcool, medicamentos, índice de massa corporal (IMC), menopausa e atividade física, por meio do Questionário Internacional de Atividade Física (IPAQ), traduzido e validado para o português. ${ }^{12}$

A prevalência de ansiedade foi estimada, bem como foi calculado seu intervalo de confiança de 95\%(IC). Para as variáveis contínuas foram calculadas médias e desvio-padrão, enquanto que para as variáveis categóricas determinaram-se as freqüências. As mulheres com e sem ansiedade foram comparadas quanto às suas características clínicas e demográficas.

Estimaram-se as odds ratios (OR) e o IC de 95\%, que foram utilizados na avaliação da associação entre a ansiedade e as demais variáveis. Consideraram-se como associações estatis- 
ticamente significativas aquelas cujo valor de $\mathrm{p}$ fosse $<0,05$. A análise estatística foi realizada utilizando-se os programas de computação Stata 8 e o epi-info 2000 versão 3.4.3.

\section{RESULTADOS}

A média de idade das mulheres incluídas no estudo ( $\mathrm{n}=749$ ) foi de 48,5 anos, ( $\mathrm{DP}=10,5)$, sendo que $41,7 \%$ delas situavam-se entre 35 e 44 anos. Predominaram as mulheres de cor branca (50,5\%), casadas $(76,0 \%)$, desempregadas
(48,5\%) e com menos de oito anos de estudo (72,6\%). Quanto à renda, 49\% referiram exercer atividade com uma renda mensal, das quais 54,9\% recebiam até um salário mínimo, (tabela 1).

Em relação aos estágios do envelhecimento ovariano, $44,3 \%$ delas estavam na pósmenopausa, A maioria (90,0\%) nunca tinha feito uso de qualquer tipo de terapia de reposição hormonal. Sobre as condições clinicas: 41\% eram hipertensas, $11 \%$ diabéticas, $17,6 \%$ exibiam a síndrome do ovário policístico, 11,4\% hiper uricemia e 10\% já tinham tido algum problema cardíaco.

Tabela1: Distribuição de freqüência das mulheres do Programa de Saúde da Família (PSF) de Pindamonhangaba, 2008.

\begin{tabular}{|c|c|c|}
\hline CARACTERÍSTICAS SÓCIO DEMOGRÁFICAS & $\mathbf{N}$ & $\%$ \\
\hline \multicolumn{3}{|l|}{ IDADE (anos) } \\
\hline $35-44$ & 307 & 41,77 \\
\hline $45-54$ & 265 & 36,05 \\
\hline $55-65$ & 163 & 22,18 \\
\hline \multicolumn{3}{|l|}{ COR } \\
\hline Branca & 376 & 50,54 \\
\hline Preta & 50 & 6,72 \\
\hline Outros & 318 & 42,74 \\
\hline \multicolumn{3}{|l|}{ RELIGIÃO } \\
\hline Católica & 488 & 65,15 \\
\hline Evangélica & 208 & 27,77 \\
\hline Outros & 53 & 7,08 \\
\hline \multicolumn{3}{|l|}{ ESTADO CIVIL } \\
\hline Casado & 540 & 76,00 \\
\hline Separada/divorciada & 109 & 15,30 \\
\hline Viúva & 62 & 8,70 \\
\hline \multicolumn{3}{|l|}{ ESCOLARIDADE (em anos) } \\
\hline 1 a 4 & 272 & 39,59 \\
\hline 5 a 8 & 227 & 33,04 \\
\hline 9 а 11 & 127 & 18,49 \\
\hline $12 \mathrm{e}+$ & 61 & 8,88 \\
\hline \multicolumn{3}{|l|}{ ATIVIDADE REMUNERDA } \\
\hline Sim & 364 & 49,06 \\
\hline Não & 378 & 50,94 \\
\hline \multicolumn{3}{|l|}{ RENDA DA MULHER(em salários mínimos) } \\
\hline Até 1SM & 200 & 54,95 \\
\hline $1 \mathrm{SM} \mathrm{e}+$ & 164 & 45,05 \\
\hline \multicolumn{3}{|l|}{ OCUPAÇÃO } \\
\hline Diarista / domestica & 134 & 35,08 \\
\hline Comércio e autônomas & 212 & 55,50 \\
\hline Aposentada / pensionista & 36 & 9,42 \\
\hline
\end{tabular}


Sobre os hábitos de vida: 35,6\% eram tabagistas, 21,9\% alcoólicas e 17,6 sedentárias; a obesidade foi observada em $32 \%$ e o sobrepeso em 69\% das participantes. Em média as mulheres apresentavam Índice de Massa Corporal (IMC) de $28, \mathrm{Kg} / \mathrm{cm}^{2}$ $(\mathrm{DP}=5,8)$

A ansiedade mostrou-se prevalente e de distribuição uniforme em 49,8\% (IC 95\%: 46,2 $53,4)$, tanto nas mulheres no estágio da transição como da pós- menopausa.

Os resultados referentes á ansiedade, identificada por meio do BAI, indicaram que o estágio leve foi de $23,6 \%$ mulheres, o moderado $16,6 \%$ e $9,6 \%$ das mulheres apresentaram a ansiedade em estágio grave com predomínio dos sintomas de irritabilidade (69,7\%), sensação de calor (53,9\%) e taquicardia (50,3\%).

Na análise bivariada observou-se que muitas variáveis tiveram associação com a ansiedade, como mostra a tabela 2, com destaque para a baixa escolaridade, atividade não remunerada e o estagio do climatério da transição menopausal.

Em relação aos hábitos de vida, destaca-se o fumo com OR de 1,73; (IC 95\%: 1,30 $-1,82 \mathrm{p}=0,001)$ e como fator protetor á ansiedade o (IMC) normal (OR 1,73; p = 0,005). As condições mórbidas apresentadas pelas mulheres não apresentaram associação estatisticamente significativa com a ansiedade.

\section{DISCUSSÃO}

O resultado deste estudo desvelou significativa prevalência de ansiedade nos estágios da transição e da pós-menopausa, que pode ser justificada pelas evidentes turbulências emocionais que os caracterizam. ${ }^{13}$

De fato, nesses estágios as mulheres se tornam mais vulneráveis e sensíveis á quadros ansiosos, segundo Barlow ${ }^{14}$, possivelmente em decorrência da redução progressiva dos estrogênios ${ }^{4,15-17}$ uma vez que esse esteróide exibe nítida ação ansiolítica. ${ }^{17}$
A ansiedade leve foi observada em 23,6\% das mulheres por nós estudadas, diferentemente dos 50\% notados por Melby et al. ${ }^{18}$ em mulheres avaliadas também pelo BAI. Essa alta prevalência foi atribuída a fatores comumente associados a esses estágios da transição e pós-menopausa, como a síndrome do “ninho vazio", aposentadoria, presença de comorbidades (hipertensão arterial e diabetes mellitus), perdas e mudanças físicas. ${ }^{9,19,20}$

Outros aspectos podem também estar envolvidos na gênese das turbulências emocionais observadas nesses estágios da transição e da pós-menopausa e que, como os outros, podem variar em intensidade e freqüência de acordo com a experiência individual de cada mulher. ${ }^{21}$

Clinicamente, a ansiedade manifesta-se por sintomas de inquietação, insegurança e tensão muscular, sendo este último uma de suas principais manifestações. ${ }^{18,21}$

Outro fator que tem sido implicado à ansiedade é a preocupação relacionada à renda, e nossos resultados confirmaram essa associação, pois das $49 \%$ de mulheres com atividade remunerada, mais da metade (54,9\%) recebiam até um salário mínimo; esse achado concorda com os constatados por La Rosa ${ }^{22}$, Melby et al. ${ }^{18}$, Maevsky et al. ${ }^{23}$ em estudos realizados em serviços públicos de saúde, ressaltando ainda que a condição de gênero e o nível sócio-econômico representaram fatores determinantes para ansiedade.

Entre os fatores sociodemográficos, constatamos maior tendência de ansiedade nas mulheres casadas (73\%), naquelas com maior escolaridade $(55,9 \%)$ e nas desempregadas (54,1\%).

Em relação ao estágio do envelhecimento ovariano, vários estudos ${ }^{5,6,24}$ já tinham observado nítida associação entre transição menopausal, pós-menopausa e ansiedade; Thomas e Brantley ${ }^{24}$, por sua vez, evidenciaram que a menopausa atua como um importante fator de risco para ansiedade. Ao comparar 
Tabela 2: Distribuição da freqüência das variáveis sócio-demograficas e clinicas e suas associações com ansiedade, número e percentagem de casos de ansiedade, odds ratio $(O R)$, intervalo de confiança (IC) 95\% e nível descritivo (valor de p) das mulheres do Programa de Saúde da Família (PSF) de Pindamonhangaba, 2008.

\begin{tabular}{|c|c|c|c|c|c|}
\hline Variável Explicativa T & Total (N) & $\begin{array}{l}\text { Ansiedade } \\
\text { Casos (\%) } \\
\end{array}$ & OR & $\begin{array}{c}\text { IC } \\
(95 \%) \\
\end{array}$ & $\begin{array}{c}\text { Nível } \\
\text { descritivo }\end{array}$ \\
\hline \multicolumn{6}{|l|}{ IDADE (anos) } \\
\hline $35-49$ & 443 & $217(59,0)$ & 0,94 & $0,81-1,09$ & 0,040 \\
\hline $50-65$ & 292 & $151(41,0)$ & & & \\
\hline \multicolumn{6}{|l|}{ COR } \\
\hline Indo européia & 376 & $187(50,4)$ & 1,09 & $0,96-1,19$ & 0,146 \\
\hline afro-brasileira & 368 & $184(49,6)$ & & & \\
\hline \multicolumn{6}{|l|}{ ESTADO CIVIL } \\
\hline Casado & 518 & $257(73,0)$ & 0,80 & $0,68-0,99$ & 0,002 \\
\hline Outros & 193 & $95(27,0)$ & & & \\
\hline \multicolumn{6}{|c|}{ ESCOLARIDADE (em anos) } \\
\hline 1 a 4 & 272 & $149(44,1)$ & 1,20 & $1,03-1,39$ & 0,005 \\
\hline 5 e + & 415 & $189(55,9)$ & & & \\
\hline \multicolumn{6}{|c|}{ ATIVIDADE REMUNERDA } \\
\hline Sim & 364 & $170(45,9)$ & 0,68 & $0,56-0,72$ & 0,001 \\
\hline Não & 378 & $200(54,1)$ & & & \\
\hline \multicolumn{6}{|c|}{ RENDA DA MULHER(em salários mínimos) } \\
\hline Ate $1 \mathrm{SM}$ & 200 & $91(53,5)$ & 1,74 & $0,75-1,97$ & 0,129 \\
\hline $1 \mathrm{SM} \mathrm{e}+$ & 164 & $79(46,5)$ & & & \\
\hline \multicolumn{6}{|c|}{ HIPERTENSÃO ARTERIAL } \\
\hline Sim & 304 & $142(38,6)$ & 1,40 & $0,78-1,95$ & 0,123 \\
\hline Não & 439 & $226(61,4)$ & & & \\
\hline \multicolumn{6}{|c|}{ DIABETES MELLITUS } \\
\hline Sim & 79 & $39(10,8)$ & 1,09 & $0,78-1,25$ & 0,466 \\
\hline Não & 650 & $323(89,2)$ & & & \\
\hline \multicolumn{6}{|l|}{ MENOPAUSA } \\
\hline Transição menopausal & 416 & $209(56,2)$ & 1,21 & $1,16-1,44$ & 0,003 \\
\hline Pós menopausa & 331 & $163(43,8)$ & & & \\
\hline \multicolumn{6}{|l|}{ FUMO } \\
\hline Sim & 152 & $71(31,7)$ & 1,73 & $1,30-1,82$ & 0,001 \\
\hline Não & 275 & $153(68,3)$ & & & \\
\hline \multicolumn{6}{|l|}{ IMC } \\
\hline Normal & 231 & $113(30,3)$ & 0,67 & $0,22-0,87$ & 0,005 \\
\hline Sobrepeso+obeso & 516 & $260(69,7)$ & & & \\
\hline \multicolumn{6}{|l|}{ USO DE HORMÔNIO } \\
\hline Sim & 74 & $35(9,4)$ & 0,34 & $0,13-0,50$ & 0,001 \\
\hline Não & 673 & $337(90,6)$ & & & \\
\hline \multicolumn{6}{|l|}{ ATIVIDADE FISICA } \\
\hline Sedentária & 132 & $73(19,6)$ & 1,11 & $0,93-1,33$ & 0,018 \\
\hline Ativa & 617 & $300(80,4)$ & & & \\
\hline
\end{tabular}


nossos resultados com os acima mencionados, observamos que a prevalência por nós detectada foi maior somente na transição menopausal.

La Rosa ${ }^{22}$ admite que a condição sócioeconômica exerça uma influência direta no determinismo da ansiedade, fato que foi confirmado em nosso estudo, onde mulheres desempregadas, apesar de terem maior escolaridade, mostraram-se ansiosas, sinalizando que o emprego e a renda, nos dias atuais, representam importantes fatores de proteção contra a ansiedade.

São escassos os estudos referentes ao uso da terapia hormonal (TH) e seu impacto sobre a ansiedade. Hays et al. ${ }^{25}$ não identificaram diferenças significativas entre usuárias e não usuárias de TH em relação à qualidade de vida, incluindo os aspectos emocionais.

O único estudo que avaliou o efeito da TH sobre a ansiedade foi o de Gambacciani et al. ${ }^{26}$, constatando redução significativa (OR:0,34; IC=95\%:0,13 - 0,50, p=0,001).

Entretanto, como nosso estudo foi realizado em uma população atendida no PSF e como a TH não é uma conduta médica comumente adotada, nosso tamanho amostral não se mostrou suficiente para se fazer conclusões apropriadas; porém, acreditamos que o estrogênio contido na TH, além de exercer diretamente ação ansiolítica, pode ainda acarretar benefícios indiretos pela redução das ondas de calor.

A prática regular da atividade física redunda em menor prevalência de ansiedade ${ }^{21} \mathrm{em}$ decorrência do aumento na secreção de beta endorfinas hipotalâmicas, que ainda melhoram o humor ${ }^{27}$, observação confirmada por Silva et al. ${ }^{7}$ Nossos resultados mostraram poucas mulheres sedentárias, pois o instrumento utilizado considera atividades ocupacionais e habituais, dificultando a análise da associação com a ansiedade.

Outro fator para o qual notamos associação com a ansiedade foi o tabagismo; de fato, apesar de somente $9,5 \%$ das parti- cipantes do nosso estudo serem fumantes, os resultados mostraram-se estaticamente significantes $(p=0,001)$, assemelhando-se aos obtidos por Lopes et al. ${ }^{28}$ que ao avaliarem 277 pacientes psiquiátricos com ansiedade e depressão constataram aumento no número de tabagistas entre os pacientes ansiosos.

As morbidades referidas na literatura como o diabetes mellitus (DM) e hipertensão arterial sistêmica $^{18,21,25}$ (HAS) não apresentam associação, porém ressaltamos que a população estudada faz parte do programa de saúde da família (PSF), e embora as mulheres apresentem as condições citadas, estas se encontram controladas, devido aos programas existentes nestas unidades como o HiperDia (Sistema de Cadastramento e Acompanhamento de Hipertensos e Diabéticos).

A variável IMC $(\mathrm{OR}=1,73 ; \mathrm{p}=0,005)$ teve associação com a ansiedade, apresentando-se como fator protetor quando as mulheres possuem um IMC normal (18,5 a 24,9 kg/m²), indo ao encontro de outros estudos que mostram uma forte relação da ansiedade com a obesidade. ${ }^{24,27}$ Há evidências de que a ansiedade e o ganho de peso no período do climatério estão relacionados com as flutuações de estrogênio comuns nesta fase, onde as situações de estresse são freqüentes e desencadeiam compulsões alimentares, bem como aumentam a síntese de cortisol, que interfere no humor provocando a irritabilidade. .,25,26 $^{2}$

Entretanto, como limitação do nosso estudo deve ser considerada a população de baixa renda, uma vez que, a avaliação da ansiedade feita por meio de um instrumento auto-referido, sem um diagnóstico clínico específico, não nos permite afirmar se o nível de ansiedade seria causa ou conseqüência das variáveis explicativas.

Do exposto, pode-se concluir que, nas mulheres que cursam os estágios da transição e pós-menopausa, é alta a prevalência de ansiedade e, entre os diversos fatores associados 
incluem-se os hábitos de vida, as condições sócio-econômicas e o estágio menopausal, com uma tendência significativa dos fatores de ordem biopsicossocial.

\section{REFERÊNCIAS:}

1. Notelovitz M. Climacteric medicine and science: a societal need. in: notelovitz m, ed. the climacteric in perspective. lancaster, 1988:19-21.

2. Soules MR, Sherman S, Parrott E, Rebar R, Santoro N, Utian W, Woods N.Executive summary: Stages of Reproductive Aging Workshop (STRAW) Climacteric. 20014 (4):267-72.

3. Gracia CR, Sammel MD, Freeman EW, Lin H, Langan E, Kapoor S, Nelson DB. Defining menopause status: creation of a new definition to identify the changes of the menopause transition. Menopause. 2005; 2(2):128-135.

4. Aldrighi JM, Aldrighi CMS, Aldrigh APS. Alterações sistêmicas no climatério. Rev Bras Méd.2002;15:21.

5. NAMS Menopause practice: a clinician's guide. USA:NAMS, 2004

6. Aldrighi JM, Alecrin IN, OliveiraPR, Shinomata HO. Tabagismo e antecipação da idade da menopausa. Rev. Assoc. Med. Bras. 2005; 51(1): 51-53.

7. Silva Filho CR, Baracat EC, Conterno LO, Haidar MA, Ferraz MB. Sintomas climatéricos e qualidade de vida: validação do questionário da saúde da mulher. Rev Saúde Pública. 2005;39:333-9

8. Freeman EW, Sammel MD, Lin H, Gracia CR, Kapoor S, Ferdousi T. The role of anxiety and hormonal changes in menopausal hot flashes. Menopause. 2005;12: 258-66.

9. Callegari C, Buttarelli M, Cromi A, Diurni M, Salvaggio F, Bolis P. Female psychopathologic profile during

\section{AGRADECIMENTOS}

À FAPESP que propiciou esta pesquisa

À Prof ${ }^{a}$ Dr $^{a}$. Nilza Nunes da Silva pelo auxilio neste artigo.

menopausal transition: a preliminary study. Maturitas. 2007;56:447-51.

10. Tangen T, Mykletun A. Depression and anxiety through the climacteric period: an epidemiological study (HUNT-II).J Psychosom Obstet Gynaecol. 2008;29(2):125-31.

11. Cunha JA. Manual da versão em português das Escalas de Beck. Casa do Psicólogo;São Paulo, 2001:171.

12. Matsudo SM, Araújo, TL, Matsudo VKR, Andrade DR, Andrade EL, Oliveira LC. Questionário internacional de atividade física (ipaq): estudo de validade e reprodutibilidade no Brasil. Rev Bras Ativ Saúde. 2001;10:5-18.

13. Souza CL. Grupos informativos sobre menopausa - informação, suporte cognitivo-comportamental e prevenção. Tese de doutorado não-publicada, Curso de Pós-graduação em Psicologia,Universidade de São Paulo, 2004.

14. Barlow DH. Anxiety and its disorders: the nature and treatment of anxiety and panic. 2nd ed. New York: the Guilford Press, 2002

15. Almeida AB; Almeida SB. Aspectos neuropsicológicos do climatério.Rev Méd Santa Casa.1996; 7(14):1449.

16. Rodríguez JAP. Endocrinología clínica: publicaciones técnicas mediterráneo. Santiago de Chile.2000

17. Yonkers KA, Bradshaw K, Halbreich U. estrogens, progestins and mood. in: steiner m, yonkers ka, ericson e (eds). mood disorders in women. london, uk: martin dunitz; 2000: 207-232.

18. Melby MK, Lock M, Kaufert P. culture and symptom reporting at menopause, Hum Reprod Update.2005; 11: 495-512. 
19. Veras AB, Rassi A, Valença AM, Nardi AE. Prevalência de transtornos depressivos e ansiosos em uma amostra ambulatorial brasileira de mulheres na menopausa. Rev Psiquiatr. 2006;28:130-4

20. Thomas JL, Brantley PJ. Factor structure of the center for epidemiologic studies depression scale in low-income women attending primary care clinics. Eur J Psychol Assess.2004; 0(2):106-15.

21. Mendito A, Cassese E, Balbi C. Climaterio e qualitá della vita. Minerva Ginecol 1999;51:83-9.

22. La Rosa J. Anxiety, sex, socioeconomic status, and birth order. Psicol. reflex. crit. 1998;11(1):59-70.

23. Maevsky EI, Peskov AB, Uchitel ML, Pogorelov AG, Saharova NY, Vihlyantseva EF, Bogdanova LA, Kondrashova MN.A succinate-based composition reverses menopausal symptoms without sex hormone replacement therapy. Adv Gerontol. 2008;21(2):298-305

24. Thomas, JL; Brantley, PJ. Factor structure of the center for epidemiologic studies depression scale in low-income women attending primary care clinics. Eur J Psychol Assess. 2004;20(2):106-15.

25. Hay AG, Bancroft J, Johnstone EC. Affective symptoms in women attending a menopause clinic. Br j Psychiatry. 1994;164:513-6

26. Gambacciani M, Ciaponi M, Cappagli B, Monteleone P, Benussi C, Bevilacqua G, et al. Effects of low-dose, continuous combined estradiol and noretisterone. Maturitas, 2003;44 (2):157-163.

27. Bossemeyer RP. Atividade física no climatério. In: Fernandes CE. Menopausa e tratamento. São Paulo: 2003: 201-9

28. Lopes FL, Nascimento I; Zin WA. Smoking and psychiatric disorders: a comorbidity survey. Braz j Med Biol Res. 2002;35(8):961-967.

Recebido em: 08/11/2008 Modificado em: 06/01/2009 Aceito em: 27/01/2009 